\title{
Immediate placement and loading of full edentulous arches in 20 periodontally compromised patients - in-line clinical case series with 3 years follow-up
}

\author{
Tran-Hung Lam ${ }^{1,2}$, Dard Michel ${ }^{3,4}$ \\ ${ }^{7}$ Faculty of Dentistry, Van Lang University, ${ }^{2}$ Elite Dental Clinic, Ho Chi Minh, Viet Nam, ${ }^{3}$ College of Dental Medicine, Columbia \\ University, New York, USA, ${ }^{4}$ Straumann Group, Basel, Switzerland
}

Purpose: These in-line clinical case series aimed to assess the cumulative implant and definitive prosthesis survival rate of full edentulous arches in periodontally compromised patients using 4 to 6 Bone Level Tapered (BLT) implants (Straumann, Basel, Switzerland) for up to 3 years of loading.

Materials and Methods: Ninety-four implants were placed in 20 patients under the full controlled principle of in-line clinical case series (14 successive days of surgery). Each subject received an immediate loaded and complete-arch provisional prosthesis 24 hours after implant surgery. The definitive prostheses were delivered within 6 to 8 weeks after implant insertion. A life table method was used to determine the implant cumulative survival rate.

Results: Our results demonstrated a cumulative implant survival rate of $98.94 \%$ up to 3 years of loading. The definitive prosthesis survival rate was $100 \%$.

Conclusions: These results support the use of BLT implants according to the ProArch concept for immediate placement and immediate loading of full edentulous arches in periodontally compromised patients. These in-line case series need to be followed by long-term clinical evaluations. (JOURNAL OF DENTAL IMPLANT RESEARCH 2021;40(3):59-65)

Key Words: Immediate placement, Immediate loading, Periodontally compromised patients

\section{INTRODUCTION}

Advanced periodontal destruction is usually associated with tooth extraction. Many studies have shown that implant can be used with success in patients treated for chronic and aggressive periodontal disease ${ }^{1,2)}$. Immediate implant placement into fresh extraction socket is considered to be a predictable and acceptable procedure ${ }^{3,4)}$. Furthermore, immediate loading of implant-supported, full-arch prostheses in the mandible or maxilla has been documented as a predictable procedure and been associated with a high level of satisfaction for patients with respect to esthetics, phonetics, and functionality. Most periodontally compromised patients with a failing dentition are candidate for immediate implant and immediate function $^{4)}$. These patients usually refuse a removable prosthesis, even provisional and request a fixed tooth prosthesis choice to resemble the natural dentition ${ }^{4,5)}$. In order to avoid a removable prosthesis and immediately restore the patient's function, a clinical protocol treating perio-

Received June 8, 2021, Revised July 2, 2021, Accepted July 7, 2021.

(c) Journal of Dental Implant Research.

This is an open access article distributed under the terms of the Creative Commons Attribution Non-Commercial License

(http://creativecommons.org/licenses/by-nc/4.0) which permits unrestricted non-commercial use, distribution, and reproduction in any medium, provided the original work is properly cited.

Correspondence to: Tran-Hung Lam, https://orcid.org/0000-0002-9598-7543

Faculty of Dentistry, Van Lang University, 69/68 Dang Thuy Tram street, District Binh Thanh, Ho Chi Minh, Viet Nam. Tel: +84(0)907773375, Fax: +(84.8)

38369716, E-mail: lam.th@vlu.edu.vn

The authors would like to thank Dr. Richard Trushkowsky for writing assistance in the preparation of the manuscript. 
dontally compromised patient with a failing dentition was developed: full arch extraction and immediate implant placement with a fixed provisional prosthesis.

Historically it was proposed to restore a full arch with at least 4 implants (All-on-4 concept, Nobel Biocare, Zurich, Switzerland). The two distal implants are tilted up to 45 degrees in order to avoid the sinus in the maxilla or the inferior alveolar nerve in the mandible; to increase anterior-posterior spread and to maximize the available bone volume. Many studies have demonstrated a high cumulative survival rate using this concept in both maxilla and mandible with an immediate loaded prosthesis in long-term follow-up ${ }^{6-9)}$.

Recently, a new concept for a standardized in-line clinical case series approach has been described for clinical investigations. This approach allows a team of clinicians to treat patients with the same indication in a consistent and successful manner ${ }^{10)}$.

Our in-line clinical case series aims to record and observe the outcomes of full edentulous arches restorations in periodontally compromised patients with 4 or 6 implants (according to the ProArch concept, Straumann, Basel, Switzerland). We combined this concept with a novel sequential bone reshaping approach following strategic extractions. The Bone Level Tapered (BLT) implants (Straumann, Basel, Switzerland) used supported a provisional, screw-retained, immediately loaded, full-arch prosthesis placed on the same day of surgery. The objective of this study was to assess the cumulative implant and definitive prosthesis survival rate for up to 3 years of loading.

\section{MATERIALS AND METHODS}

Patients presenting with hopeless teeth and foreseen to be treated with a full arch rehabilitation procedure were selected from the medical records of the Elite Dental clinic (Ho Chi Minh city, Viet Nam) on their willingness to receive an immediately loaded and complete-arch provisional prosthesis during the 24 hours after implant surgery and their availability to be treated from April 4th, 2016 to April 18th, 2016. These patients agreed on the type of rehabilitation and to the related documentation process which corresponds to a daily practice to a daily practice approach at Elite Dental clinic. This collection of cases do not need to be submitted to an Institutional Review Board (IRB) as it is purely observational and not experimental.

This timely organization presents the advantage to keep the surgical team at a high level of focus and calibration ensuring the highest quality of treatment for the patients and adding value to the standard treatment. 20 patients (11 males and 9 females aged from 40 to 79 years-old) were operated at the mandible (11 cases) or at the maxilla (12 cases) under the principle of in-line clinical case series ${ }^{10)}$.

In total 94 BLT implants with an SLActive surface (Institut Straumann, Basel, Switzerland) were inserted supporting 23 prostheses.

As for a standard procedure it was paid attention that the subjects present a bone volume allowing the placement of at least 4 implants of minimum $8 \mathrm{~mm}$ length in either healed or extracted sites, that their general health allows for implant dentistry treatment and that they present an acceptable oral hygiene.

The definitive prostheses were delivered within 6 to 8 weeks after implant insertion. A life table method corresponding to the standard procedure of patient's documentation was used to determine implant cumulative survival rate. The technical procedure was defined ahead of the surgical/prosthetic phase and deviations thoroughly recorded as normally performed in regular implant dentistry treatments.

\section{Implant surgery}

All patients were treated with free hand surgery. The surgical procedures were performed under local anesthesia with Septocanine (Epinephrine 1:100,000) (Septodont, Saint-Maur-Des-Fossés, France). For the maxilla, the anesthesia was injected to block the bilateral infraorbitalis nerve, bilateral paltinus nerve, incisive nerve and also the vestibulum. For the mandible, the anesthesia was injected to block the bilateral alveolar nerve and also the vestibule and lingual mucosa.

In failing dentition patients, a novel sequential bone reshaping approach following strategic extractions was implemented. Instead of extraction of all the failing teeth (Fig. 1A, 1B) prior to implant placement, we performed 

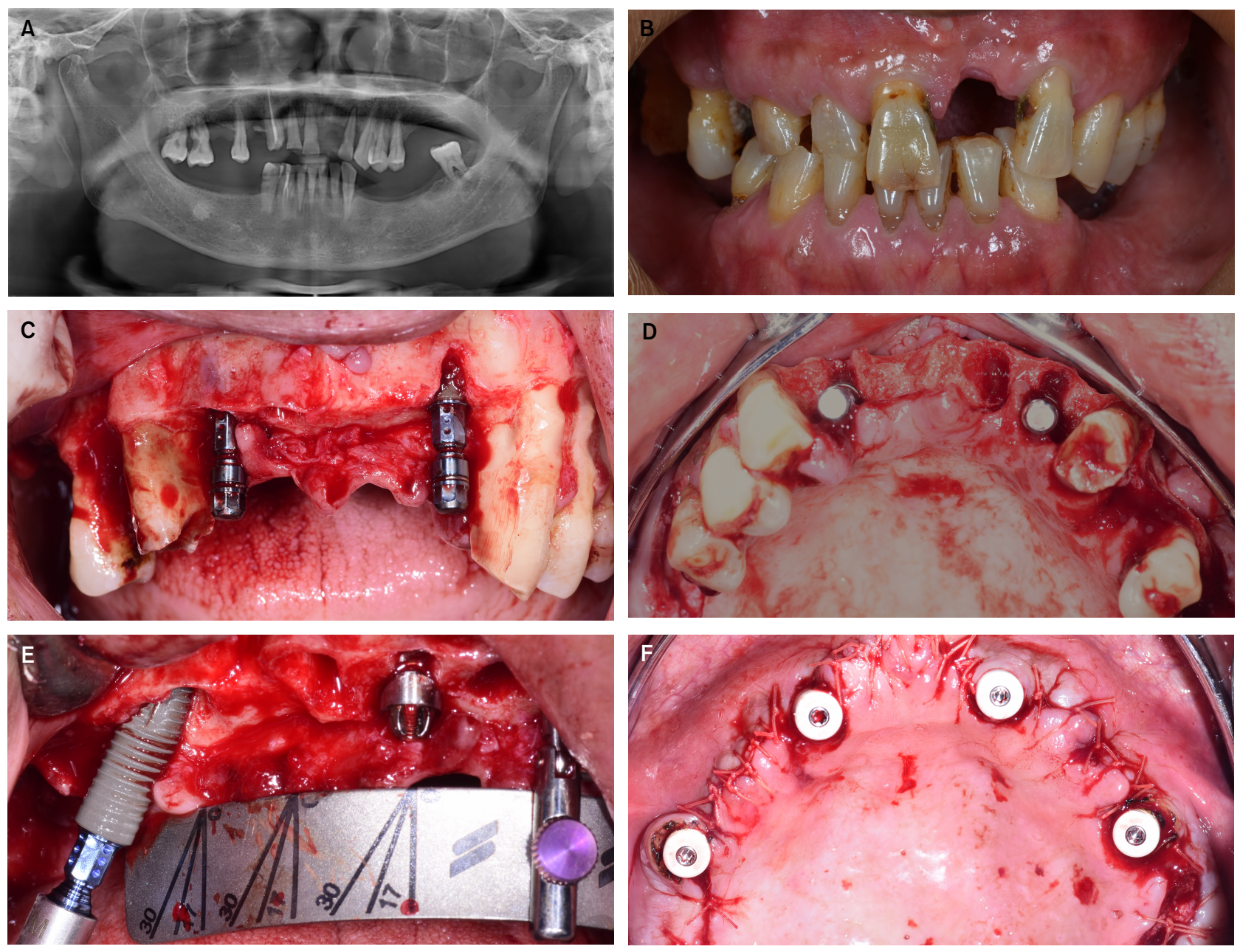

Fig. 1. Surgical protocol with a novel sequential bone reshaping approach following strategic extractions. (A) Panorex before surgery. (B) Failing dentition in the maxilla. (C) Strategic extraction of two lateral incisors and implant placement in these position. (D) Occlusal view of two anterior implant. (E) Extraction, bone reshaping and placing of posterior implant using ProArch guide. (F) Connection of screw abutment and healing cap.

the strategic extraction, usually two lateral incisors (Fig. 1C, 1D). Then alveolectomy was performed using the round bur to smoothen the alveolar bone. The bone drilling sequence was followed and then the implant was placed in this position. The extraction of failing teeth next to this position was performed and followed by bone reshaping using the round bur.

Two distal implants were tilted in order to avoid the sinus or the inferior nerve. If the visibility of the ventral sinus wall was insufficient, a small opening was made to the sinus in order to visualize the anterior wall of the sinus using a periodontal probe. In the mandible, the mental foramen was exposed to place the posterior implant as close as possible without violating the nerve. The tilted implants were positioned anterior to the maxillary sinus or to the mental foramen using ProArch guide (Institut Straumann, Basel, Switzerland) (Fig. 1E) to reduce the cantilever length. This guide allowed the implant positioning and the inclination of tilted implants (17 or 30 degree). Once the implant had been placed, the screw abutment was torqued at $35 \mathrm{Ncm}$ for the re-alignment of the prosthetic axis (Fig. 1F). The selection of the abutment height was depending of the soft tissue thickness.

In case of having enough bone volume in the posterior area, the implants were straight. The drill sequences for BLT implants were followed using the manufacturer's manual (Institut Straumann, Basel, Switzerland). After the 2.2 diameter dill, the 2.8 or 3.5 or 4.1 diameter drill were used to enlarge the osteotomy depending the implant diameter. In case of hard bone, the thread cutting, and 

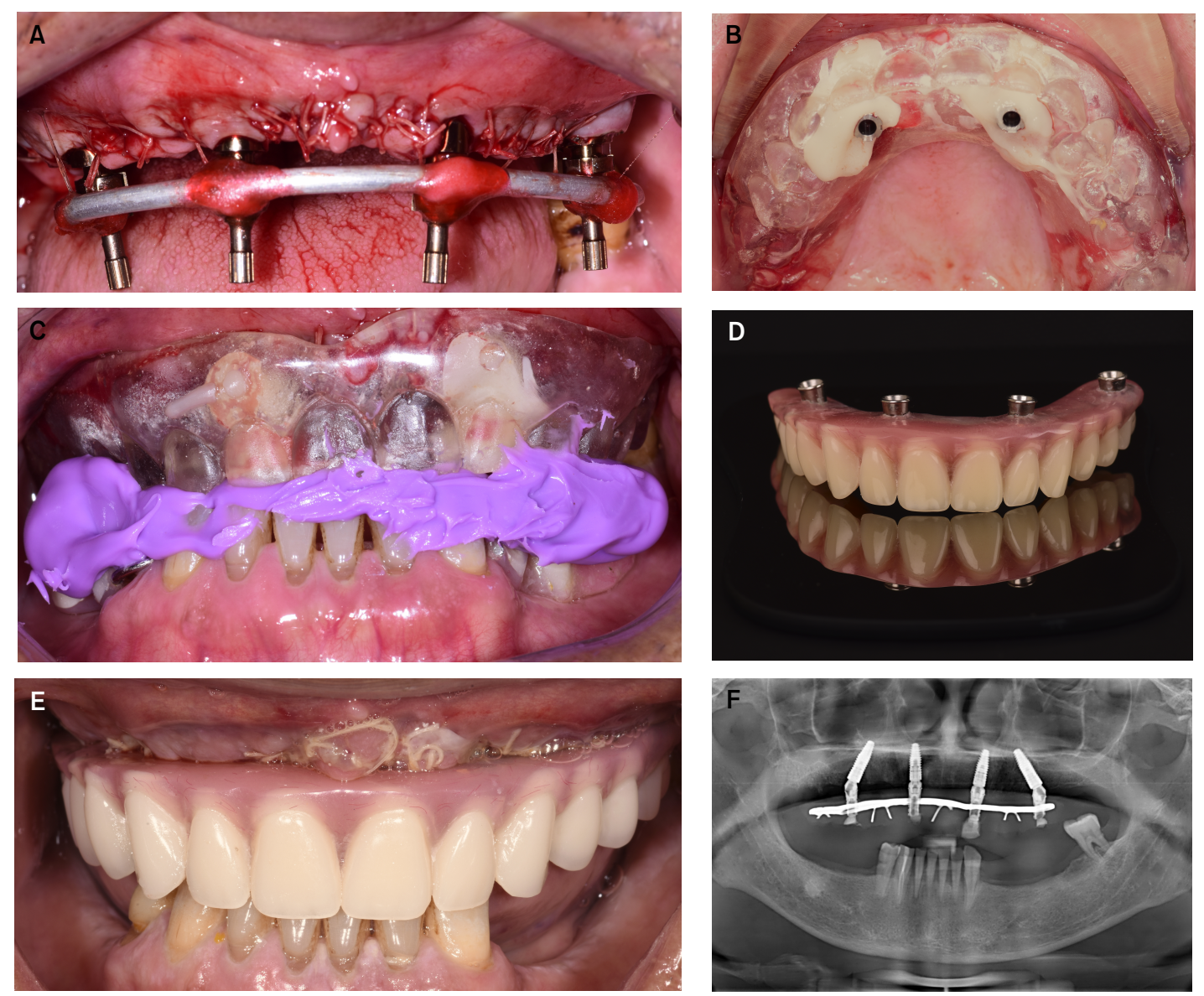

Fig. 2. Prosthetic protocol for immediate loading. (A) Splinting of impression copings by means of a metal bar and Pattern resin. (B) Bite registration using a prefabricated index stent and two titanium temporary cylinders connecting to the transparent index stent. (C) Bite registration with Occlufast. (D) Immediate provisional prosthesis. (E) Delivery of immediate provisional prosthesis. (F) Panorex after surgery.

profiling drill were applied. The final torque of implant insertion was measured using torque wrench (Institut Straumann, Basel, Switzerland).

The following post-surgical medications were given: Augmentine $1 \mathrm{~g}$ ( 2 per day), Medrol $16 \mathrm{mg}$ (only one in the morning), Efferalgan $500 \mathrm{mg}$ (3 per day).

\section{Prosthetic protocol}

Impression copings were screwed onto the abutment and splinted together by means of a metal bar and acrylic resin (Pattern resin, GC, Asia) (Fig. 2A). The impression was performed using polyvinyl siloxane (Honigum, DMG, Germany) with the double mix technique. The bite registration was performed using a prefabricated index stent with two titanium temporary cylinders connected and Occlufast (Zermark, Badia Polesine, Italy) (Fig. 2B, $2 \mathrm{C}$ ). Both information was sent to the laboratory to pro- duce a complete arch provisional prosthesis (Fig. 2D). The provisional was delivered during $24 \mathrm{~h}$ with a torque value of $15 \mathrm{Ncm}$ (Fig. 2E) and performed a check-up with panoramic $X$ ray (Fig. $2 F$ ).

After $6 \sim 8$ weeks, the definitive prosthesis was initiated (Fig. 3). This prosthesis consisted of a milled cobalt-chrome framework obtained by CAD/CAM procedure (Milling machines 5X-200, ARUM Dentistry, Korea) with layering ceramic (Ceramco, Dentply, Germany) or acrylic resin with denture teeth (IvoBase, Ivoclar Vivadent, Europe) depending on patient's desires. Only 1 clinician performed all restorations.

\section{Survival criteria}

Survival criteria used in our study are the following:

1. An implant was classified surviving when it remained in the jaw and was restored with the final 

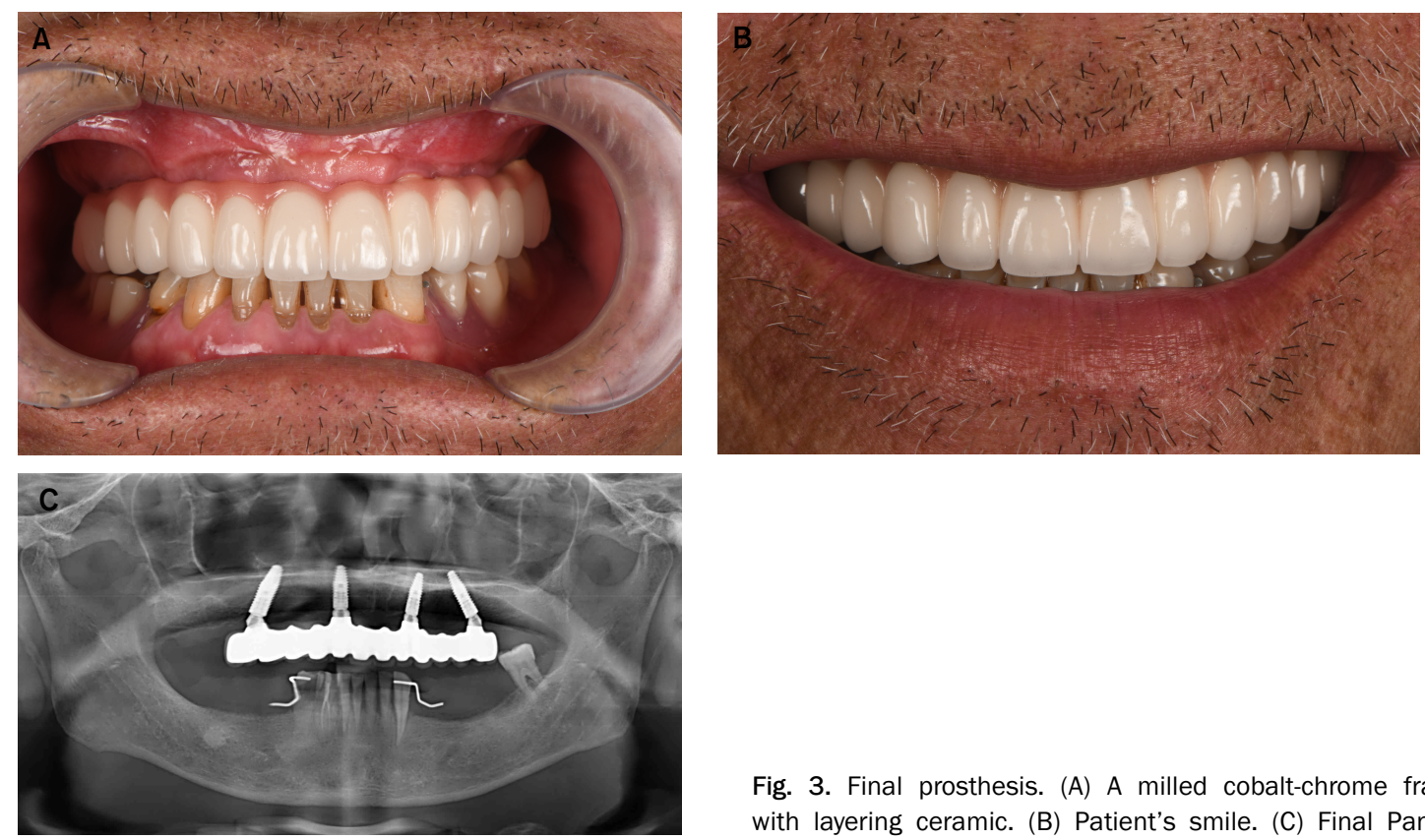

Fig. 3. Final prosthesis. (A) A milled cobalt-chrome framework with layering ceramic. (B) Patient's smile. (C) Final Panorex.

Table 1. Torque value of implant insertion

\begin{tabular}{lcc}
\hline $\mathrm{Ncm}$ & $\mathrm{n}$ & $\%$ \\
\hline$<35$ & 17 & 18.09 \\
35 & 49 & 52.13 \\
$>35$ & 28 & 29.78 \\
Total & 94 & 100 \\
\hline
\end{tabular}

prosthesis.

2. A final prosthesis was classified surviving when it was stable and remained functioning on patient's mouth.

The clinical follow up was performed between April 2016 and April 2019 to assess the cumulative implant and definitive prosthesis survival rate.

\section{RESULTS}

In these in-line clinical case series, $94 \mathrm{TiZr}$ Bone Level Tapered implants (48 straight, 46 tilted) with a hydrophilic surface (SLActive surface) were inserted in 20 patients. Fifty implants have been placed at the maxilla and 44 in the mandible. Three patients were treated in both jaws. Most of the prosthesis were supported by 4 implants, only 2 prostheses in the maxilla were supported by 6 implants. Most of the implants $(52.13 \%)$ were inserted with a minimum of $35 \mathrm{Ncm}$ torque. Only 18.09\% of the implants were seated at a torque of $<35 \mathrm{Ncm}$. The
Table 2. Implant size (BLT Straumann)

\begin{tabular}{crr}
\hline $\begin{array}{c}\text { Implant } \\
\text { diameter } \\
(\mathrm{mm})\end{array}$ & Implant length $(\mathrm{mm})$ & $\mathrm{n}$ \\
\hline 3.3 & 8 & 0 \\
& 10 & 8 \\
& 12 & 6 \\
4.1 & 14 & 4 \\
& 8 & 4 \\
& 10 & 30 \\
& 12 & 20 \\
4.8 & 14 & 12 \\
& 8 & 6 \\
& 10 & 2 \\
& 12 & 2 \\
Total & 14 & 0 \\
& & 94 \\
\hline
\end{tabular}

average insertion torque reached $35.5 \mathrm{Ncm}$ (Table 1).

Implant distribution according to implant diameter and length is described in Table 2. The most used implant was $4.1 \mathrm{~mm}$ diameter and $10 \mathrm{~mm}$ length. The cumulative survival rate is described in Table 3.

Our novel sequential bone reshaping approach following strategic extractions facilitates the implant positioning and the assessment of the prosthetic space for the final prosthesis (Fig. 1). No surgical complications during or immediately after surgery were reported.

The definitive prosthesis survival rate was $100 \%$ after 
Table 3. Cumulative survival rate

\begin{tabular}{lccccc}
\hline $\begin{array}{c}\text { Time of interval } \\
\text { (months) }\end{array}$ & $\begin{array}{c}\text { Implants at start } \\
\text { of interval }\end{array}$ & $\begin{array}{c}\text { Dropouts during } \\
\text { interval }\end{array}$ & $\begin{array}{c}\text { Implants under } \\
\text { risk }\end{array}$ & $\begin{array}{c}\text { Failure during } \\
\text { interval }\end{array}$ & Survival rate (\%) \\
\hline $0 \sim 3$ & 94 & 0 & 94 & 1 & 98.94 \\
$3 \sim 6$ & 93 & 0 & 93 & 0 & 100 \\
$6 \sim 12$ & 93 & 0 & 93 & 0 & 100 \\
$12 \sim 18$ & 93 & 0 & 93 & 0 & 100 \\
$18 \sim 24$ & 93 & 0 & 93 & 0 & 100 \\
$24 \sim 30$ & 93 & 0 & 93 & 0 & 98.94 \\
$30 \sim 36$ & 93 & 0 & 93 & 0 & 98.94 \\
\hline
\end{tabular}

3 years of loading.

\section{DISCUSSION}

Our study is the first follow-up using BLT implants to support a full arch fixed prosthesis in periodontally compromised patients. In our in line clinical case series, the cumulative survival rate was $98.94 \%$ and the definitive prosthesis survival rate was $100 \%$ for up to 3 years of loading. Only 1 implant failed in the first 2 months of loading before the definitive prosthesis has been performed. Most of the prosthesis was supported by 4 implants and 2 prostheses on the maxilla were supported by 6 implants. There were 46/94 implants that have been placed tilted. The purpose of placing tilted implants is to maximize the remaining bone volume, reduce the cantilever length and avoid the bone augmentation procedure $^{6,8)}$. There is no difference in term of bone loss around implant and clinical outcomes between straight and tilted implants to support the full edentulous $\operatorname{arch}^{7,8)}$.

Our original sequential bone reshaping procedure following strategic extractions was described in this in-line clinical case series. This procedure facilitates the implant positioning and the assessment of the prosthetic space for the final prosthesis. Periodontally compromised patients lose not only teeth but also the bone and soft tissue. That's the reason why an FP-3 (fixed prosthesis with pink material in Misch's classification ${ }^{11)}$ ) is usually indicated for final prosthesis. This kind of prosthesis needs enough prosthetic height space and the transitional line should be more apical in compare to the smile line.

Most of studies have recommended that an insertion torque from 30 to $40 \mathrm{Ncm}$ is required to perform immediate loading for single-tooth implant ${ }^{12-14)}$. In our clinical case series, the average insertion torque reached 35.5
Ncm. However, there was 17 implants (18.09\%) (Table 1) having torque value only $25 \mathrm{Ncm}$. These implants still successfully support the provisional bridges immediately loaded under precautions: all the implants are splinted with a rigid provisional prosthesis; avoid cantilever for provisional prosthesis and the final prosthesis was performed at least 2 months after implant placement. This approach is favorised by the use of an implant manufactured in a Ti-Zr alloy presenting an SLA hydrophilic surface. These two elements contributing to the regulation of mesenchymal stem cell differentiation and maturation $^{15)}$ and eventually to an optimal osseointegration even under immediate loading conditions ${ }^{16)}$.

Up to 3 years of loading, our results demonstrated a cumulative implant survival rate of $98.94 \%$ and the definitive prosthesis survival rate was $100 \%$. Previously published studies reporting implant survival rate using the All-on-4 concept is similar to the result of our study ${ }^{4,5)}$. In a recent study of 10 to 18 years follow-up, Malo and his co-authors ${ }^{9)}$ have demonstrated that implant cumulative survival rate was $94.7 \%$ and prosthetic success rate was $99.2 \%$. These results confirm this treatment concept is predictable and safe in mid-term and long-term outcome.

In our clinical case series, all periodontally compromised patients have been restored with a fixed rehabilitation which encompasses the whole procedure from removal of hopeless teeth, immediate placement of four to six implants and im $\neg$ mediate loading of the implants with a provisional prosthesis. It also includes the treatment planning steps before surgery and afterwards when converting the temporary bridge to the final full-arch prosthesis. The full arch restoration with 4 to 6 implants lessens the number of surgeries as bone grafting procedure is not performed and diminishes therefore 
treatment cost for the patient ${ }^{17)}$. The psychologic implications and the natural refusal for a removable prosthesis of periodontally compromised patients lead them to delay the decision to extract their hopeless teeth, risking even more bone loss. Immediate function significantly increases the patient's acceptance for total treatment, therefore, reduces the complexity of the procedure. When restoring periodontally compromised patients, implant-supported prosthesis treatment should be considered to achieve potentially oral health-related quality of life compare to removable dentures ${ }^{18)}$.

\section{CONCLUSION}

These results support the use of BLT implants according to the ProArch concept for immediate placement and immediate loading to restore full arch in periodontally compromised patients. This observational study in form of in-line clinical case series demonstrated a cumulative implant survival rate of $98.94 \%$ and a definitive prosthesis survival rate of $100 \%$ up to 3 years of loading. The reported positive clinical outcomes pave the way for long term clinical evaluations.

\section{ORCID}

Tran-Hung Lam, https://orcid.org/0000-0002-9598-7543

Dard Michel, https://orcid.org/0000-0002-9107-996X

\section{REFERENCES}

1. Baelum V, Ellegaard B. Implant survival in periodontally compromised patients. J Periodontol 2004;75:1404-12.

2. Wennström JL, Ekestubbe A, Gröndahl K, Karlsson S, Lindhe J. Oral rehabilitation with implant-supported fixed partial dentures in periodontitis-susceptible subjects. A 5-year prospective study. J Clin Periodontol 2004;31:713-24.

3. Schwartz-Arad D, Chaushu G. The ways and wherefores of immediate placement of implants into fresh extraction sites: A literature review. J Periodontol 1997;68:915-23.
4. Alves CC, Correia AR, Neves M. Immediate implants and immediate loading in periodontally compromised patients-a 3-year prospective clinical study. Int J Periodontics Restorative Dent 2010;30:447-55.

5. Malo P, de Araujo Nobre M, Rangert B. Implants placed in immediate function in periodontally compromised sites: a five-year retrospective and one-year prospective study. J Prosthet Dent 2007;97(6 Suppl):S86-S95.

6. Chan MH, Holmes C. Contemporary "All-on-4" concept. Dent Clin North Am 2015;59:421-70.

7. Soto-Penaloza D, Zaragozí-Alonso R, Penarrocha-Diago M, Penarrocha-Diago M. The all-on-four treatment concept: Systematic review. J Clin Exp Dent 2017;9:474-88.

8. Patzelt SB, Bahat O, Reynolds MA, Strub JR. The all-on-four treatment concept: a systematic review. Clin Implant Dent Relat Res 2014;16:836-55.

9. Maló P, de Araújo Nobre M, Lopes A, Ferro A, Botto J. The All-on-4 treatment concept for the rehabilitation of the completely edentulous mandible: A longitudinal study with 10 to 18 years of follow-up. Clin Implant Dent Relat Res 2019;21: 565-77.

10. Dard M. The standardized in-line clinical case series: a new concept for real-world evidence in dentistry. J Dent Oral Health 2017;3:1-6.

11. Misch CE. Contemporary implant dentistry, 3th ed. St. Louis, Missouri: Mosby Elsevier; 2007:92-103.

12. Hui E, Chow J, Li D, Liu J, Wat P, Law H. Immediate provisional for single-tooth implant replacement with Branemark system: preliminary report. Clin Implant Dent Relat Res 2001;3:79-86.

13. Wegscheider WA. Immediate loading of single-tooth implants in the anterior maxilla: Preliminary results after one year. Clin Oral Implants Res 2003;14:180-7.

14. Tettamanti L, Andrisani C, Bassi MA, Vinci R, Silvestre-Rangil J, Tagliabue A. Immediate loading implants: review of the critical aspects. Oral Implantol 2017;10:129-39.

15. Boyan BD, Cheng A, Olivares-Navarrete R, Schwartz Z. Implant surface design regulates mesenchymal stem cell differentiation and maturation. Adv Dent Res 2016;28:10-7.

16. Stavropoulos A, Cochran D, Obrecht M, Pippenger BE, Dard M. Effect of osteotomy preparation on osseointegration of immediately loaded, tapered dental implants. Adv Dent Res 2016; 28:34-41.

17. Balshi TJ, Wolfinger GJ, Slauch RW, Balshi SF. A retrospective analysis of 800 Brånemark System implants following the All-on-Four ${ }^{\mathrm{TM}}$ protocol. J Prosthodont 2014;23:83-88.

18. Fischer KR, Lindner I, FickI S. Implant treatment in periodontally compromised subjects-quality of life and patient satisfaction. Clin Oral Investig 2016;20:697-702. 